\title{
Fast mean and variance computation of the diffuse sound transmission through finite-sized thick and layered wall and floor systems
}

\author{
Carolina Decraene ${ }^{\mathrm{a}, *}$, Arne Dijckmans ${ }^{\mathrm{a}}$, Edwin P.B. Reynders ${ }^{\mathrm{a}}$ \\ ${ }^{a} K U$ Leuven, Department of Civil Engineering, Kasteelpark Arenberg 40, 3001 Leuven, Belgium
}

\begin{abstract}
A method is developed for computing the mean and variance of the diffuse field sound transmission loss of finite-sized layered wall and floor systems that consist of solid, fluid and/or poroelastic layers. This is achieved by coupling a transfer matrix model of the wall or floor to statistical energy analysis subsystem models of the adjacent room volumes. The modal behavior of the wall is approximately accounted for by projecting the wall displacement onto a set of sinusoidal lateral basis functions. This hybrid modal transfer matrix-statistical energy analysis method is validated on multiple wall systems: a thin steel plate, a polymethyl methacrylate panel, a thick brick wall, a sandwich panel, a double-leaf wall with poro-elastic material in the cavity, and a double glazing. The predictions are compared with experimental data and with results obtained using alternative prediction methods such as the transfer matrix method with spatial windowing, the hybrid wave based-transfer matrix method, and the hybrid finite element-statistical energy analysis method. These comparisons confirm the prediction accuracy of the proposed method and the computational efficiency against the conventional hybrid finite element-statistical energy analysis method.
\end{abstract}

Keywords: sound insulation, transfer matrix method, statistical energy analysis, hybrid deterministic-stochastic models

\section{Introduction}

Layered wall and floor systems are frequently applied in order to achieve a high thermal insulation and/or a high sound insulation with a relatively low weight. Examples are double walls with decoupled wall leafs, sandwich panels, floors with floating screeds, double and triple glazing, etc. At high frequencies, the airborne or impact sound transmission through such layered systems can be computed with high accuracy using the transfer matrix method (TMM) $[1,2,3]$. It is then assumed that the wall or floor is of infinite lateral extent and that the adjacent sound fields can be modeled statistically, as diffuse fields. This enables computing the ensemble mean of the diffuse sound transmission through each solid, fluid and/or poro-elastic layer analytically in the frequency-wavenumber domain.

However, the conventional TMM has two disadvantages. Firstly, the assumption of an infinite wall may lead to important prediction errors at low and medium frequencies. The influence of edge

\footnotetext{
${ }^{*}$ Corresponding author. Tel.: +32 (0) 16379124.

Email address: carolina.decraene@kuleuven.be (Carolina Decraene)
} 
diffraction, which is important below the critical frequency, may be accounted for by spatial windowing $[4,5]$, but then the numerical integration of the plane-wave transmission over all possible angles of incidence for obtaining the mean sound transmission loss results in a large computation time when implemented in a straightforward way. Different analytic revisions of the finite size correction attempt to reduce the computation time $[6,7]$. The assumption of similar lateral dimensions speeds up the calculations very significantly [8]. However, with spatial windowing the modal behavior of the wall is still neglected. An alternative has therefore been developed [9, 10], in which the wall displacement is projected onto a set of sinusoidal lateral basis functions, such that the wall impedance at a given frequency and for a given lateral basis function (hence lateral wavenumber) can be computed using the TMM. This approach accounts approximately for modal wall behavior when the boundary conditions are simply supported: if the radiation impedance of the sound field to the chosen basis functions can be computed with high accuracy, then the results are accurate for thin plates as the mode shapes of the plate correspond to the imposed basis functions. For most thick and layered systems, sinusoidal lateral basis functions also result in accurate transmission loss predictions. The predictions will be less accurate if the damping is low and the stiffness's of the different layers differ strongly from each other, as in the case of double glazing or double walls without absorbent material in the cavity $[9,10]$. The approximate modal version of the TMM will be referred to as mTMM in the remainder of this article. As shown later in the present paper, the accuracy of the ensemble mean transmission loss predictions obtained with mTMM depends critically on the chosen method for approximating the modal sound radiation impedances.

A second disadvantage of conventional TMM concerns the diffuse field assumption in both the sending and receiving room. A diffuse sound model is a statistical model representing an ensemble of rooms that are identical except for wave scattering at the room boundaries or by objects within the room: this wave scattering is modeled statistically in a maximum entropy sense, i.e., the uncertainty induced by the wave scattering is maximized $[11,12]$. Only the ensemble mean of the sound transmission loss can be computed by the different variations of the TMM. Other statistics that are inherent to the diffuse sound field assumption, such as the variance of the sound transmission loss, can not be obtained.

There exists an alternative vibro-acoustic modeling approach for which this variance can be computed: the hybrid finite element-statistical energy analysis (FE-SEA) approach [13, 14, 15]. Using the diffuse field reciprocity $[12,16]$, the hybrid framework enables modeling the rooms of the overall room-wall-room system to carry a diffuse field (as in statistical energy analysis, SEA), while the wall is modeled deterministically, with finite element analysis. Since both the mean and the variance of the transmission loss can be computed [14], the uncertainty on the transmission loss predictions that is inherent to the assumption of diffuse sound fields in the rooms can be assessed [15]. However, at high frequencies a conventional FE-SEA model can be computationally demanding as a very fine mesh is required for modeling the wall with finite elements.

In the present paper, the aforementioned limitations of the FE-SEA method and the mTMM method are overcome by developing a hybrid mTMM-SEA method. This method can be viewed as a fast alternative for the hybrid FE-SEA method. The computational efficiency is achieved by replacing the finite element model of a thick or layered wall in the hybrid FE-SEA model by a semi-analytical modal transfer matrix model. This still enables to account for the diffraction effects at the wall boundaries and (approximately) the modal behavior of the wall, while reducing the computational effort substantially. However, such replacement is only possible when the wall satisfies the following assumptions: it is baffled, rectangular and consists of homogeneous layers. 


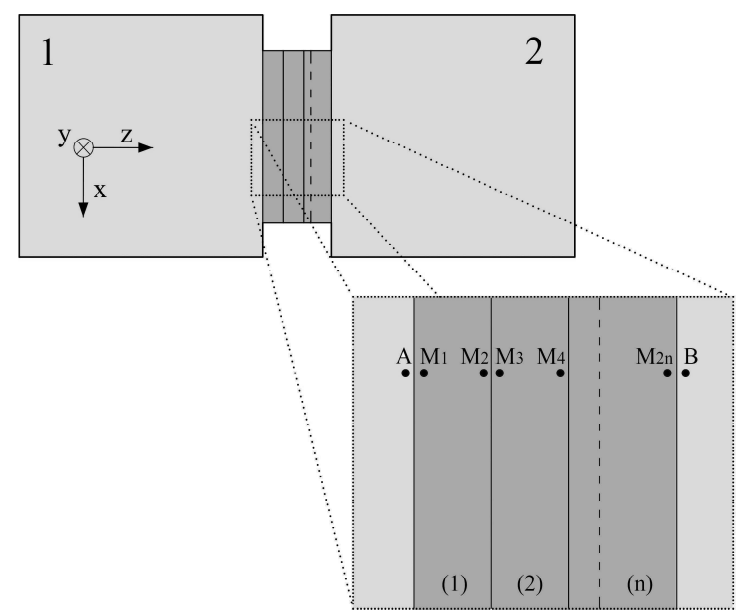

Figure 1: Multilayered system.

From an mTMM point of view, casting the mTMM within the hybrid framework enables to make it more general and robust. The detailed contributions of the hybrid mTMM-SEA framework that is presented in this paper are (1) with respect to the TMM and related approaches (1a) that the variance, which is inherent to the diffuse field models of the sound fields can be computed at low computational cost, (1b) that cross-modal coupling is accounted for, while keeping a similar computation cost as in the conventional mTMM and (1c) that numerical difficulties in evaluating the plane-wave transmission, related to grazing incidence, are avoided; and (2) with respect to the hybrid FE-SEA approach: a substantial increase in computational efficiency when the system is simple enough to be modeled with mTMM.

The remainder of this article is organized as follows. The theory is summarized in section 2 . Section 2.1 introduces the hybrid deterministic-SEA approach to sound transmission modeling in general. Sections 2.2 and 2.3 elaborate the computation of the dynamic stiffness matrices of respectively the deterministic part (wall) and the stochastic parts (rooms). In the former the connection is made with the transfer matrix method. In the latter, the approach for computing the modal sound radiation impedance in the hybrid method is compared with the conventional approaches employed in mTMM. Subsequently, the performance of the hybrid mTMM-SEA approach is investigated in detail for six walls with increasing complexity, by comparing the predicted sound insulation values with results of other hybrid approaches and with measured values. The considered wall types are a steel plate (section 3.1), a polymethyl methacrylate panel (section 3.2), a brick wall (section 3.3), a sandwich panel (section 3.4), a double-leaf wall with poro-elastic material in the cavity (section 3.5), and finally a double glazing (section 3.6). The conclusions are presented in section 4 .

\section{A hybrid modal transfer matrix - statistical energy analysis framework}

\subsection{Modeling strategy}

Throughout this article, a room-wall-room system is considered, where the rooms carry a diffuse wave field and the wall a deterministic wave field (cfr. Fig. 1). The out-of-plane displacement $u_{\mathrm{z}}$ 
of the partition wall is approximated using a finite set of $N_{\mathrm{m}}$ global basis functions $\phi$ (e.g. the invacuo modes) and corresponding generalized coordinates $q$. When the wall is thin, the transverse displacement of the wall in position $\mathbf{x}=(x, y)$ at frequency $\omega$ is independent of the $\mathrm{z}$-coordinate:

$$
u_{\mathrm{z}}(\mathbf{x}, \omega) \approx \sum_{p=1}^{N_{\mathrm{m}}} \phi_{p}(\mathbf{x}) q_{p}(\omega)
$$

For a thick wall, the decomposition is performed for each of both outer surfaces A and B:

$$
u_{\mathrm{z}}\left(\mathbf{x}, z_{\mathrm{A}}, \omega\right) \approx \sum_{p=1}^{N_{\mathrm{m}}} \phi_{p}\left(\mathbf{x}, z_{\mathrm{A}}\right) q_{p \mathrm{~A}}(\omega) \quad \text { and } \quad u_{\mathrm{z}}\left(\mathbf{x}, z_{\mathrm{B}}, \omega\right) \approx \sum_{p=1}^{N_{\mathrm{m}}} \phi_{p}\left(\mathbf{x}, z_{\mathrm{B}}\right) q_{p \mathrm{~B}}(\omega)
$$

All generalized response degrees of freedom (DOFs) are collected in an amplitude vector $\mathbf{q}(\omega) \in$ $\mathbb{C}^{N_{\text {dof }}}$, so that the time-domain response is given by $\operatorname{Re}\left(\mathbf{q} \mathrm{e}^{\mathrm{i} \omega t}\right)$, with i the imaginary unit. Similarly, the corresponding generalized harmonic loads are collected in the load amplitude vector $\mathbf{f}(\omega) \in$ $\mathbb{C}^{N_{\text {dof }}}$. Note that, when the wall is modeled as thin, then $N_{\text {dof }}=N_{\mathrm{m}}$, but when it is thick, then $N_{\text {dof }}=2 N_{\mathrm{m}}$. Since q contains all (generalized) interface degrees of freedom between the wall and the rooms, the equations of motion of the whole system (room-wall-room) can be written as

$$
\mathbf{D q}=\mathbf{f},
$$

with $\mathbf{D} \in \mathbb{C}^{N_{\text {dof }} \times N_{\text {dof }}}$ the dynamic stiffness matrix at frequency $\omega$. D may be decomposed as the sum of the dynamic stiffness matrix of the wall, denoted as $\mathbf{D}_{\mathrm{d}}$ (subscript $\mathrm{d}$ stands for deterministic), and the dynamic stiffness matrices of the rooms, denoted as $\mathbf{D}_{1}$ and $\mathbf{D}_{2}$ :

$$
\mathbf{D}=\mathbf{D}_{\mathrm{d}}+\mathbf{D}_{1}+\mathbf{D}_{2}
$$

Since the rooms are assumed to carry a diffuse field in this work, they are modeled as the random subsystems in the overall room-wall-room system. The dynamic stiffness matrix of each acoustic room volume $k$ is decomposed as

$$
\mathbf{D}_{k}=\mathbf{D}_{\text {dir }}^{(k)}+\mathbf{D}_{\text {rev }}^{(k)}, \quad k=1,2
$$

where $\mathbf{D}_{\text {dir }}^{(k)}$ denotes the mean of the subsystem's dynamic stiffness matrix $\mathbf{D}_{\text {dir }}^{(k)}:=\mathrm{E}\left[\mathbf{D}_{k}\right]$. This term describes the part of the response of subsystem $k$ containing outgoing waves only. It is the limiting response of the acoustic volume to the displacements of the deterministic boundary that would be observed at that boundary when the extent of the subsystem would be increased. It can be computed as the direct field receptance matrix of the room at room-wall interface $[12,17]$. $\mathbf{D}_{\text {rev }}^{(k)}$ represents the response of the reverberant field in the subsystem, caused by the presence of random wave scattering boundaries or objects. With this decomposition, the equations of motion for a random subsystem are

$$
\mathbf{D}_{\text {dir }}^{(k)} \mathbf{q}=\mathbf{f}_{k}+\mathbf{f}_{\mathrm{rev}}^{(k)}
$$

where the reverberant forces are defined as $\mathbf{f}_{\text {rev }}^{(k)}:=-\mathbf{D}_{\text {rev }}^{(k)} \mathbf{q}$, and $\mathbf{f}_{k}$ denotes the sum of the loads applied to subsystem $k$ at its DOFs. The overall equations of motion (3) become

$$
\mathbf{D}_{\mathrm{tot}} \mathbf{q}=\mathbf{f}+\mathbf{f}_{\mathrm{rev}}^{(1)}+\mathbf{f}_{\mathrm{rev}}^{(2)}
$$


where $\mathbf{D}_{\text {tot }}:=\mathbf{D}_{\mathrm{d}}+\sum_{k=1}^{2} \mathbf{D}_{\text {dir }}^{(k)}$ is a purely deterministic matrix. When a diffuse field acts in both rooms, the reverberant forces $\mathbf{f}_{\mathrm{rev}}^{(k)}$ are related to $\mathbf{D}_{\text {dir }}^{(k)}$ through the diffuse field reciprocity relationship [12]:

$$
\mathrm{E}\left[\mathbf{f}_{\mathrm{rev}}^{(k)} \mathbf{f}_{\mathrm{rev}}^{(s)}\right]=\delta_{k s} \frac{4 \hat{E}_{k}}{\omega \pi n_{k}} \operatorname{Im}\left(\mathbf{D}_{\mathrm{dir}}^{(k)}\right)
$$

with $n_{k}$ the modal density of subsystem $k$ and $\delta_{k s}$ the Kronecker delta. Note that the hat symbol is employed here as shorthand notation for ensemble mean. From this equation, it is possible to obtain the mean time-averaged total energy $\hat{E}_{k}$ of room $k$ from a stationary power balance which involves the other random subsystems as well as the deterministic master system, assuming the fields are statistically independent of each other. For the case where the external loading acts solely on the random subsystems (rooms), this reads [13]:

$$
\omega\left(\eta_{k}+\hat{\eta}_{\mathrm{d}, k}\right) \hat{E}_{k}+\sum_{j=1}^{2} \omega \hat{\eta}_{k j} n_{k}\left(\frac{\hat{E}_{k}}{n_{k}}-\frac{\hat{E}_{j}}{n_{j}}\right)=\hat{P}_{k}, \quad k=1,2 .
$$

In this expression, $\eta_{k}$ is the damping loss factor of subsystem $k, P_{k}$ the power input from external loading injected directly into the diffuse field of this subsystem, and

$$
\begin{aligned}
\hat{\eta}_{\mathrm{d}, k} & =\frac{2}{\omega \pi n_{k}} \sum_{r, s} \operatorname{Im}\left(D_{\mathrm{d}, r s}\right)\left(\mathbf{D}_{\mathrm{tot}}^{-1} \operatorname{Im}\left(\mathbf{D}_{\mathrm{dir}}^{(k)}\right) \mathbf{D}_{\mathrm{tot}}^{-\mathrm{H}}\right)_{r s} \\
\hat{\eta}_{k j} & =\frac{2}{\omega \pi n_{k}} \sum_{r, s} \operatorname{Im}\left(D_{\mathrm{dir}, r s}^{(k)}\right)\left(\mathbf{D}_{\mathrm{tot}}^{-1} \operatorname{Im}\left(\mathbf{D}_{\mathrm{dir}}^{(j)}\right) \mathbf{D}_{\mathrm{tot}}^{-\mathrm{H}}\right)_{r s}
\end{aligned}
$$

where the superscript $\mathrm{H}$ denotes Hermitian transpose and the integer subscripts $r, s$ select an element in row $r$ and column $s$ of a matrix. If the wall provides sufficient sound insulation, $\hat{P}_{k}$ can be approximated as the ensemble mean of the power input into a hard-walled room. It can be noted that the power balance equation (9) has formally the same structure as in conventional SEA. Therefore the factors $\hat{\eta}_{k j}$ can be interpreted as coupling loss factors, and (11) provides a rigorous way to compute their ensemble mean, even when the overall system is partly deterministic. In a similar manner, (10) enables to rigorously estimate the power that is lost by energy dissipation in the wall. The computation of the dynamic stiffness matrix of the wall, $\mathbf{D}_{\mathrm{d}}$, and of the direct field dynamic stiffness matrices of the rooms as seen from the baffled wall, $\mathbf{D}_{\text {dir }}^{(1)}$ and $\mathbf{D}_{\text {dir }}^{(2)}$, will be discussed in sections 2.2 and 2.3, respectively. By solving for the mean coupling loss factor $\hat{\eta}_{12}$ between the two subsystems, the mean sound transmission coefficient $\hat{\tau}_{12}$ can be obtained from [5]

$$
\hat{\tau}_{12}=\hat{\eta}_{12} \frac{4 \omega V_{1}}{c_{a} S}
$$

where $S$ denotes the surface area of the wall, $V_{1}$ the volume of the sending room, and $c_{a}$ the speed of sound.

An important advantage of the general deterministic-SEA framework described above, is that not only the mean, but also the variance of the transmission loss which is inherent in the diffuse field models of the rooms, can be obtained. It was demonstrated by Langley and Cotoni [14] that this variance can be computed by a first-order perturbation analysis of the power balance (9); 
Reynders et al. [15] then made the connection with the variance of the sound transmission loss. This variance can be computed in closed form as follows:

$$
\sigma_{\tau}^{2}=\operatorname{Relcov}\left[C_{12}, C_{12}\right] \hat{\tau}_{12}^{2},
$$

with

$$
\begin{aligned}
\operatorname{Rel} \operatorname{cov}\left[C_{12}, C_{12}\right]= & \sum_{k}\left(\frac{a_{k}}{\pi m_{k}^{\prime}}\right)\left\{\frac{\operatorname{Tr}\left(\tilde{\mathbf{D}}_{\text {dir }}^{(k)} \mathbf{G}^{(2)}\right) \operatorname{Tr}\left(\mathbf{H}_{k} \mathbf{G}^{(2)}\right)+\operatorname{Tr}\left(\tilde{\mathbf{D}}_{\mathrm{dir}}^{(k)} \mathbf{G}^{(2)} \mathbf{H}_{k} \mathbf{G}^{(2)}\right)}{\operatorname{Tr}\left(\tilde{\mathbf{D}}_{\text {dir }}^{(1)} \mathbf{G}^{(2)}\right) \operatorname{Tr}\left(\tilde{\mathbf{D}}_{\mathrm{dir}}^{(k)} \mathbf{G}^{(2)}\right)}\right\} \\
& +\left\{\frac{\operatorname{Tr}\left(\tilde{\mathbf{D}}_{\text {dir }}^{(1)} \mathbf{G}^{(2)} \tilde{\mathbf{D}}_{\text {dir }}^{(1)} \mathbf{G}^{(2)}\right)}{\operatorname{Tr}\left(\tilde{\mathbf{D}}_{\mathrm{dir}}^{(1)} \mathbf{G}^{(2)}\right) \operatorname{Tr}\left(\tilde{\mathbf{D}}_{\mathrm{dir}}^{(1)} \mathbf{G}^{(2)}\right)}\right\},
\end{aligned}
$$

where the tilde $(\sim)$ represents the imaginary part of a matrix, the diffuse field correction factor $a_{k}$ is equal to 1 as only area couplings are considered in this work, Tr is the trace matrix, and with

$$
\begin{aligned}
& \mathbf{G}^{(2)}=\left(\frac{4 a_{2}}{\omega \pi}\right) \mathbf{D}_{\mathrm{tot}}^{-1} \operatorname{Re}\left\{\mathbf{D}_{\mathrm{dir}}^{(2)}\right\} \mathbf{D}_{\mathrm{tot}}^{-\mathrm{H}}, \\
& \mathbf{H}_{k}=4 \tilde{\mathbf{D}}_{\mathrm{dir}}^{(1)} \mathbf{D}_{\mathrm{tot}}^{-1} \tilde{\mathbf{D}}_{\mathrm{dir}}^{(k)} \mathbf{D}_{\mathrm{tot}}^{-\mathrm{H}} \tilde{\mathbf{D}}_{\mathrm{dir}}^{(1)}+\tilde{\mathbf{D}}_{\mathrm{dir}}^{(1)} \delta_{k 1}-2 \delta_{k 1} \mathrm{i} \tilde{\mathbf{D}}_{\mathrm{dir}}^{(1)} \mathbf{D}_{\mathrm{tot}}^{-1} \tilde{\mathbf{D}}_{\mathrm{dir}}^{(1)}+2 \delta_{k 1} \mathrm{i} \tilde{\mathbf{D}}_{\mathrm{dir}}^{(1)} \mathbf{D}_{\mathrm{tot}}^{-\mathrm{H}} \tilde{\mathbf{D}}_{\mathrm{dir}}^{(1)} .
\end{aligned}
$$

The effective modal overlap factor $m_{k}^{\prime}$, which appears in equation (14), is defined as [18]:

$$
m_{k}^{\prime}=\omega \eta_{k} n_{k},
$$

in which the damping loss factors $\eta_{k}$ and modal densities $n_{k}$ of the rooms can be estimated as $[5,19]$ :

$$
\eta_{k}=\frac{4.4 \pi}{\omega T_{k}} \quad \text { and } \quad n_{k}=\frac{\omega^{2} V_{k}}{2 \pi^{2} c_{a}^{3}},
$$

with $T_{k}$ the reverberation time of room $k$ and $V_{k}$ its volume. When the mean and variance sound transmission are known, then the mean $\hat{R}$ and variance $\sigma_{R}^{2}$ of the sound transmission loss $R$ follow from

$$
\hat{R}=10 \log \frac{1}{\hat{\tau}_{12}}
$$

and

$$
\sigma_{R}^{2}=\left(\frac{-10}{\ln (10) \hat{\tau}_{12}}\right)^{2} \sigma_{\tau}^{2}
$$

2.2. Computing the deterministic dynamic stiffness matrix from finite wall impedances using the transfer matrix method

In the sound transmission prediction framework that was outlined in the previous section, the dynamic stiffness matrix of the wall $\mathbf{D}_{\mathrm{d}}$ is needed in modal coordinates. When the wall is a thin simply supported plate of dimensions $L_{x} \times L_{y} \times t$, a natural choice for the basis functions in (1) are the exact plate mode shapes, which are [5, Sec. 5.7.2]

$$
\phi_{p}(\mathbf{x})=\sin \left(\frac{p_{x} \pi x}{L_{x}}\right) \sin \left(\frac{p_{y} \pi y}{L_{y}}\right),
$$


where $p_{x}$ and $p_{y}$ denote the integer number of half wavelengths in the $\mathrm{x}$ and $\mathrm{y}$ directions, respectively, of mode $p$. The elements of the dynamic stiffness matrix are then

$$
D_{\mathrm{d}, p}(\omega)=\frac{\rho t L_{x} L_{y}}{4}\left(-\omega^{2}+\omega_{p}^{2}\left(1+\mathrm{i} \eta_{\mathrm{p}}\right)\right) \delta_{p q}
$$

where $\rho$ denotes the density of the plate material, i the imaginary unit, $\delta_{p q}$ the Kronecker delta, $\eta_{\mathrm{p}}$ the internal loss factor of the plate, and $\omega_{p}$ the natural frequency of mode $p$ :

$$
\omega_{p}=\sqrt{\frac{D}{\rho t}}\left(k_{p x}^{2}+k_{p y}^{2}\right), \quad D:=\frac{E t^{3}}{12\left(1-\nu^{2}\right)}, \quad k_{p x}:=\frac{p_{x} \pi}{L_{x}}, \quad k_{p y}:=\frac{p_{y} \pi}{L_{y}}
$$

with $E$ the Young's modulus of the plate and $\nu$ the Poisson's ratio.

For more complex walls or floors, the dynamic stiffness matrix $\mathbf{D}_{\mathrm{d}}$ will need to be computed in a different way. Of course, general numerical approaches such as the finite element method may be used [13, 20,21], yet for thick and multilayered walls and floors, a much more efficient approach is possible, as demonstrated in what follows.

The transfer matrix method is a semi-analytical method for modeling the sound transmission through infinite layered wall and floor systems $[1,2,3]$. The layers may consist of solid, fluid or porous material. They are assumed to be infinite in the $\mathrm{x}$ - and $\mathrm{y}$-directions. The degrees of freedom of each layer are the complex amplitudes of the velocity and stress fields at both boundary surfaces. These degrees of freedom of layer $l$ are collected in the vectors $\ell\left(M_{2 l-1}\right)$ and $\boldsymbol{\ell}\left(M_{2 l}\right)$ (cfr. Fig. 1). For every layer $l$, a transfer matrix is constructed that describes the two-dimensional (x-z) wave propagation in the layer by relating, for a particular wavenumber $k_{x}$ and a particular frequency $\omega$, all degrees of freedom in $\ell\left(M_{2 l-1}\right)$ and $\ell\left(M_{2 l}\right)$ to each other. The derivation of the transfer matrix of a fluid layer is straightforward; see, e.g., [19]. The expressions become more complex for solid layers and for poroelastic layers (Biot theory) - see [22] and [3, Ch. 11], respectively - yet they can still be obtained in closed form. As an alternative, the porous layer can also be modeled as an equivalent fluid, i.e., as a fluid layer with complex and frequency-dependent material properties. A detailed comparison between the full Biot model and an equivalent fluid model for a specific wall interlayer can be found further on in this paper (§3.4). The continuity conditions between different layers are described by interface matrices. By using the transfer and interface matrices in the correct order, a global transfer matrix $\mathbf{G} \in \mathbb{C}^{(R-2) \times R}$ is composed which describes the behavior of the layered system. When the degrees of freedom of the overall layered system are collected in the vector $\ell_{\mathrm{G}} \in \mathbb{C}^{R \times 1}$,

$$
\ell_{\mathrm{G}}=\left[\begin{array}{lllllll}
p_{\mathrm{A}}\left(k_{x}, \omega\right) & v_{\mathrm{zA}}\left(k_{x}, \omega\right) & L\left(M_{2}\right) & L\left(M_{4}\right) & \ldots & p_{\mathrm{B}}\left(k_{x}, \omega\right) & v_{\mathrm{zB}}\left(k_{x}, \omega\right)
\end{array}\right]^{\mathrm{T}},
$$

one has that

$$
\mathbf{G} \ell_{\mathrm{G}}=\mathbf{0} .
$$

The relation between the pressures $p_{\mathrm{A}}, p_{\mathrm{B}}$ and normal velocities $v_{\mathrm{zA}}, v_{\mathrm{zB}}$ at both sides of the multilayered structure is obtained by computing the mechanical impedance matrix $\mathbf{Z}\left(k_{x}, \omega\right) \in \mathbb{C}^{2 \times 2}$

$$
\left[\begin{array}{ll}
Z_{11}\left(k_{x}, \omega\right) & Z_{12}\left(k_{x}, \omega\right) \\
Z_{21}\left(k_{x}, \omega\right) & Z_{22}\left(k_{x}, \omega\right)
\end{array}\right]\left[\begin{array}{l}
v_{\mathrm{zA}}\left(k_{x}, \omega\right) \\
v_{\mathrm{zB}}\left(k_{x}, \omega\right)
\end{array}\right]=\left[\begin{array}{l}
p_{\mathrm{A}}\left(k_{x}, \omega\right) \\
p_{\mathrm{B}}\left(k_{x}, \omega\right)
\end{array}\right]
$$


from the global transfer matrix $\mathbf{G}[23]$ :

$$
\begin{aligned}
& Z_{11}\left(k_{x}, \omega\right)=-\frac{\left|\mathbf{G}_{1, R}\right|}{\left|\mathbf{G}_{2, R}\right|}, \quad Z_{12}\left(k_{x}, \omega\right)=\frac{\left|\mathbf{G}_{1,2}\right|}{\left|\mathbf{G}_{2, R}\right|}, \\
& Z_{21}\left(k_{x}, \omega\right)=-\frac{\left|\mathbf{G}_{R-1, R}\right|}{\left|\mathbf{G}_{2, R}\right|}, \quad Z_{22}\left(k_{x}, \omega\right)=-\frac{\left|\mathbf{G}_{2, R-1}\right|}{\left|\mathbf{G}_{2, R}\right|}
\end{aligned}
$$

where $\left|\mathbf{G}_{r, s}\right|$ is the determinant of $\mathbf{G}$, in which the columns $r$ and $s$ are eliminated.

So far, the modeling of infinite thick and layered walls has been discussed. For finite walls, the normal displacement $u_{\mathrm{z}}(\mathbf{x}, z, \omega)$ can be approximated using a Ritz approach, i.e., by means of a finite set of shape functions $\phi_{p}(\mathbf{x})$ that satisfy the boundary conditions (e.g., simply supported):

$$
u_{\mathrm{z}}(\mathbf{x}, z, \omega) \approx \sum_{p=1}^{N_{\mathrm{m}}} \phi_{p}(\mathbf{x}) q_{p}(z, \omega)
$$

The plate velocities and the pressures at both sides of the wall can then be written as $[9,10]$

$$
\left[\begin{array}{l}
v_{\mathrm{zA}}(\mathbf{x}, \omega) \\
v_{\mathrm{zB}}(\mathbf{x}, \omega)
\end{array}\right]=\sum_{p}\left[\begin{array}{l}
V_{\mathrm{zAp}}(\omega) \\
V_{\mathrm{zBp}}(\omega)
\end{array}\right] \phi_{p}(\mathbf{x}) \quad \text { and } \quad\left[\begin{array}{l}
p_{\mathrm{A}}(\mathbf{x}, \omega) \\
p_{\mathrm{B}}(\mathbf{x}, \omega)
\end{array}\right]=\sum_{p}\left[\begin{array}{l}
P_{\mathrm{Ap}}(\omega) \\
P_{\mathrm{Bp}}(\omega)
\end{array}\right] \phi_{p}(\mathbf{x})
$$

When $\phi_{p}(\mathbf{x})$ is chosen as in (21), it corresponds to a bending mode shape of a rectangular thin plate with modal wavenumber

$$
k_{p}=\sqrt{k_{p x}^{2}+k_{p y}^{2}} .
$$

The modal response of this homogeneous thin plate is obtained by imposing the modal wavenumber as trace wavenumber in an infinite plate model. Furthermore, since the basis functions $\phi_{p}(\mathbf{x})$ are orthogonal to each other, the following equation is valid:

$$
\int_{0}^{L_{x}} \int_{0}^{L_{y}} \phi_{p}(\mathbf{x}) \phi_{q}(\mathbf{x}) \mathrm{d} x \mathrm{~d} y=\frac{L_{x} L_{y}}{4} \delta_{p q}
$$

Using this property when combining equations (26) and (28), one has the following exact relationship for a finite homogeneous thin plate:

$$
\left[\begin{array}{ll}
Z_{11}\left(k_{p}, \omega\right) & Z_{12}\left(k_{p}, \omega\right) \\
Z_{21}\left(k_{p}, \omega\right) & Z_{22}\left(k_{p}, \omega\right)
\end{array}\right]\left[\begin{array}{l}
V_{\mathrm{zAp}}(\omega) \\
V_{\mathrm{zBp}}(\omega)
\end{array}\right]=\left[\begin{array}{l}
P_{\mathrm{Ap}}(\omega) \\
P_{\mathrm{Bp}}(\omega)
\end{array}\right]
$$

This implies that (1) the impedances of the wall in the chosen generalized coordinates (i.e., simply supported thin plate modal coordinates) can be computed independently for each basis function, and (2) the impedance matrix between both sides of a finite wall in generalized coordinates can be evaluated as the impedance matrix of the corresponding infinite wall, evaluated for the wavenumber $k_{p}$ of the basis function $p$. In order to relate the mechanical impedance matrix to the dynamic stiffness matrix of the structure in generalized coordinates, the transformation from modal velocity to modal displacement and the transformation from modal pressure to modal force are elaborated here:

$$
q_{p}=\frac{d V_{z p}}{\mathrm{~d} t}=\mathrm{i} \omega V_{z p}
$$




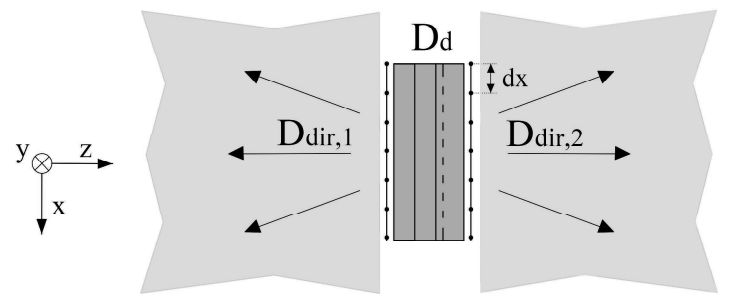

Figure 2: Illustration of the direct field dynamic stiffness matrix for the case of a transmission suite.

$$
f_{p}=\int_{0}^{L_{x}} \int_{0}^{L_{y}} p(\mathbf{x}) \phi_{p}(\mathbf{x}) \mathrm{d} x \mathrm{~d} y=\int_{0}^{L_{x}} \int_{0}^{L_{y}} P_{p} \phi_{p}^{2}(\mathbf{x}) \mathrm{d} x \mathrm{~d} y=P_{p} \frac{L_{x} L_{y}}{4}
$$

Thus, the deterministic dynamic stiffness matrix follows from:

$$
\mathbf{D}_{\mathrm{d}, \mathrm{p}}(\omega)=\frac{\mathrm{i} \omega L_{x} L_{y}}{4} \mathbf{Z}\left(k_{p}, \omega\right)
$$

Eq. (34) is exact for a simply supported thin plate. Its validity for other wall types depends on the validity of approximation (27), which assumes that only waves with in-plane wavenumbers $k_{p}$ can propagate through the wall. This assumption is generally valid unless the dynamic stiffness's of the different layers differ strongly from each other and the damping is low, e.g., when stiff solid layers are coupled via an intermediate air layer [9]. In this case however, an elegant and efficient solution is possible by modeling the air layer as a hard-walled cavity. This will be demonstrated further on, in section 3.6 and in Appendix A.

\subsection{Computing the direct field dynamic stiffness matrix of the rooms}

In order to apply the diffuse field reciprocity relationship, the acoustic dynamic stiffness matrix should be determined for a baffled interface that radiates into an acoustic halfspace (cfr. Fig. 2 ). The surface pressure $p(\mathbf{x})$ due to the displacement $u_{z}(\mathbf{x})$ of the structural-acoustic interface is determined by the Rayleigh integral [24]

$$
p(\mathbf{x})=\int_{S} g\left(\mathbf{x}-\mathbf{x}^{\prime}\right) u_{z}\left(\mathbf{x}^{\prime}\right) \mathrm{d} \mathbf{x}^{\prime} \quad \text { with } \quad g(\mathbf{x})=-\frac{\rho_{a} \omega^{2} \mathrm{e}^{-\mathrm{i} k_{a}|\mathbf{x}|}}{2 \pi|\mathbf{x}|}
$$

where the terms $\rho_{a}$ and $k_{a}$ represent the air density and the acoustic wavenumber, respectively. In order to evaluate this expression for an arbitrary displacement field, the interface between the room and the wall can be covered by a grid of points $\mathbf{x}_{n}$ with equal grid spacings in both directions. The displacements are then decomposed into a set of generalized degrees of freedom $a_{n}(n=1,2, \ldots, N)$ and shape functions $\varphi_{n}=\varphi\left(\mathbf{x}-\mathbf{x}_{n}\right)(n=1,2, \ldots, N)$ :

$$
u_{z}(\mathbf{x})=\sum_{n=1}^{N} a_{n} \varphi\left(\mathbf{x}-\mathbf{x}_{n}\right)
$$


In the wavelet approach [25], the shape functions $\varphi\left(\mathbf{x}-\mathbf{x}_{n}\right)$ are chosen to be jinc functions, jinc $(x)=\mathrm{J}_{1}(x) / x$ with $\mathrm{J}_{1}(x)$ the Bessel function of the first kind and order 1 , so that

$$
\varphi\left(\mathbf{x}-\mathbf{x}_{n}\right)=\frac{2 \mathrm{~J}_{1}\left(k_{s} r\right)}{k_{s} r}, \quad \text { where } r=\left|\mathbf{x}-\mathbf{x}_{n}\right| \text { and } k_{s}=\sqrt{2} \pi / d x
$$

where $d x$ denotes the grid spacing. Equations (36) and (37) are substituted into equation (35). In this way the Rayleigh integral can be further elaborated using the specific properties of the jinc function and the pressure is expressed in terms of the generalized coordinates $a_{n}$ from (36). The generalized force $f_{m}$ acting on a degree of freedom $a_{m}$ due to this pressure becomes [25]

$$
\begin{aligned}
f_{m} & =\int_{S} p(\mathbf{x}) \varphi_{m}(\mathbf{x}) \mathrm{d} \mathbf{x} \\
& =\sum_{n} D_{m n} a_{n}
\end{aligned}
$$

with

$$
\begin{aligned}
& D_{m n}=D\left(r_{m n}, \omega\right)=\frac{\mathrm{i} 8 \pi \omega \rho_{a} c_{a} k_{a}^{2}}{k_{s}^{4}}\left(\operatorname{sinc}\left(k_{a} r_{m n}\right)+\mathrm{i} g\left(k_{a} r_{m n}\right)\right), \quad r_{m n}=\left|\mathbf{x}_{m}-\mathbf{x}_{n}\right| \\
& g(z)=\frac{\cos z-1}{z}+\frac{2}{z} \sum_{k=1}^{\infty} \mathrm{J}_{2 k+1}\left(z k_{s} / k_{a}\right)
\end{aligned}
$$

This formulation allows an efficient computation of the acoustic dynamic stiffness matrix. However, the obtained matrix still needs to be projected onto the generalized coordinates $q$, used in the hybrid mTMM-SEA approach. This can be done as follows:

$$
D_{\text {dir }, p q}(\omega)=\mathbf{a}_{p}^{\mathrm{T}} \mathbf{D}(\omega) \mathbf{a}_{q}, \quad \text { with } \mathbf{a}_{p}=\frac{\pi}{2} \phi_{p}\left(\mathbf{x}_{n}\right)
$$

where $p$ and $q$ define two modes, considered in the calculation. The basis functions $\phi_{p}$ were defined earlier in equation (21). The resulting $\mathbf{D}_{\text {dir }}$ is a fully populated matrix, which takes cross-modal coupling into account.

A faster calculation of the direct field dynamic stiffness matrix is possible, yet at the expense of accuracy. Different approaches for computing the radiation impedance $z_{p}$, normalized to the characteristic impedance of the fluid medium $Z_{c}$, have been presented previously. Leppington et al. [26] simplify the exact quadruple integral, which results from the combination of Eqs. (35) and (38), to a more efficient expression by using polar coordinates, which is then approximated with asymptotic estimates for the real part of the normalized radiation impedance. However, automated numerical integration of the Leppington formula with adaptive integration routines faces numerical difficulties. This has been solved by Davy et al. [27], who reformulated the Leppington expression, and extended this to include the imaginary part of the radiation impedance. Besides the numerical evaluation of the exact integral expressions, they have also elaborated approximate formulae for both the real and imaginary part of the radiation impedance. Because these approximations do not require numerical integration, the radiation impedance can be calculated at very low computational cost. The diagional terms $D_{\mathrm{dir}, p p}$ are then derived from $z_{p}$ in the following way:

$$
D_{\text {dir }, p p}=\frac{\mathrm{i} \omega L_{x} L_{y}}{4} Z_{c} z_{p}
$$




\begin{tabular}{|l|c|c|c|c|}
\hline Time $[\mathrm{s}]$ & wavelets & Lepp. appr. & Davy int. & Davy appr. \\
\hline PMMA panel & 230.7 & 22.2 & 10752.9 & 4.7 \\
\hline
\end{tabular}

Table 1: Computation time for $\mathbf{D}_{\text {dir }}$ using the wavelet approach of Langley, the approximate asymptotic formulae of Leppington, the integral formulation of Davy and the approximate formulae of Davy.

In order to investigate the differences between the various discussed approaches to obtain $\mathbf{D}_{\text {dir }}$, the sound transmission loss of a polymethyl methacrylate (PMMA) panel has been plotted using the wavelet approach of Langley, the asymptotic estimates of Leppington et al. and the integral formulation and approximate formulae, proposed by Davy et al., embedded within the framework of the mTMM-SEA approach. Note that the wavelet approach is normally used in hybrid FE-SEA, while the other approaches are normally used in mTMM. The geometric and material properties of the PMMA panel will be detailed in section 3.2. Figure 3 and table 1 show respectively the results in 1/48 octave bands and the computation costs corresponding to the different methods for evaluating $\mathbf{D}_{\text {dir }}$. Only the wavelet approach takes cross-modal coupling into account, so the results obtained with this approach are the most accurate ones. There are clear differences with the other approaches until ca. $500 \mathrm{~Hz}$, which implies that cross-modal coupling influences the transmission loss below $500 \mathrm{~Hz}$ in the present case. In particular, it can be seen that the approximations of Leppington et al. and Davy et al. and the exact numerical integration of Davy et al. do not capture the anti-resonances at $59.2 \mathrm{~Hz}$ and $79.9 \mathrm{~Hz}$. Note that the conventional mTMM cannot take cross-modal coupling into account [10], while this becomes possible within the hybrid mTMM-SEA framework presented here. The exact numerical integration approach of Davy et al. agrees best with the wavelet approach. Larger differences are noted for the approximate integration of Davy et al. and for the asymptotic estimates of Leppington. Such differences are indeed expected mainly below coincidence [27]. However, the increase in computational efficiency is very substantial, so application of the approximate expressions of Davy et al. or Leppington et al. render the mTMMSEA approach much more efficient but less accurate. For the sake of accuracy, the wavelet approach is adopted throughout the remainder of this work.

\section{Validation examples}

In this section, the proposed mTMM-SEA approach is validated on wall systems of increasing complexity. The predicted sound transmission loss values are compared against experimental results and alternative prediction results. All calculations were performed on a computer with a 2.70 GHz Intel Core i7 processor and 16 GB RAM.

In all but one example (the double-leaf wall of Section 3.5), the experimental results have been obtained at the KU Leuven Laboratory of Acoustics. The rooms in the transmission suite of the KU Leuven Laboratory of Acoustics have a volume of $87 \mathrm{~m}^{3}$. Wall systems are either tested in a large transmission opening $(3.25 \mathrm{~m} \times 2.95 \mathrm{~m})$ or a small transmission opening $(1.25 \mathrm{~m} \times 1.5 \mathrm{~m})$. The measurements have been performed in accordance with the ISO 10140 standard [28], except that the sound pressure levels were integrated over $1 / 48$-octave bands instead of $1 / 3$-octave bands in order to have a higher frequency resolution. With the exception of the sandwich panel (section 3.4), the damping loss factors of the walls were determined experimentally in $1 / 3$ octave bands from structural reverberation time tests when the test sample was mounted in the vertical transmission opening, in accordance with ISO 10848-1 [29]. The resulting values are listed in Table 2. In all 


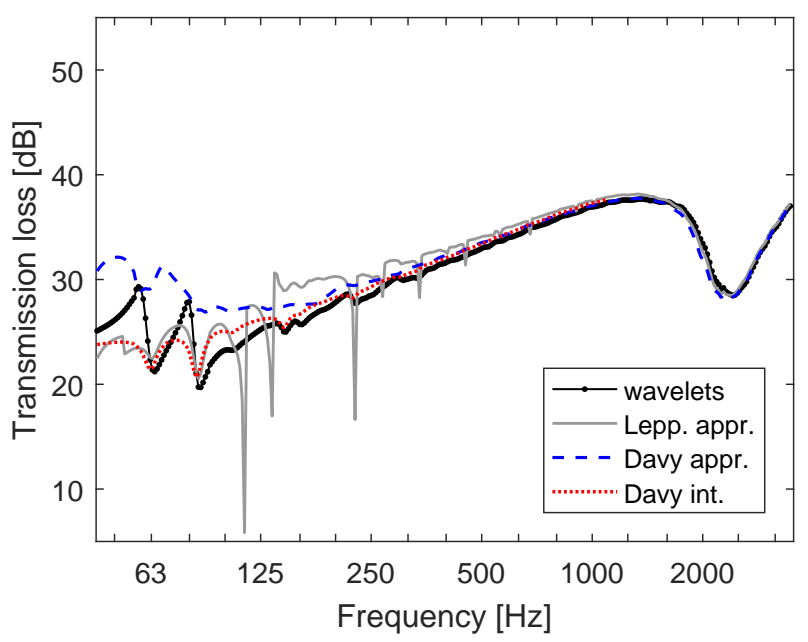

Figure 3: Harmonic sound transmission loss of a PMMA panel, calculated with the mTMM-SEA approach, in which $\mathbf{D}_{\text {dir }}$ is derived from the wavelet approach of Langley, from the approximate asymptotic formulae of Leppington, from the integral formulation of Davy or from the approximate formulae of Davy.

simulations, the reverberation time $T$, the air density $\rho_{a}$ and the sound speed $c_{a}$ in the rooms are taken to be $1.5 \mathrm{~s}, 1.2 \frac{\mathrm{kg}}{\mathrm{m}^{3}}$ and $343 \frac{\mathrm{m}}{\mathrm{s}}$, respectively. Note that in the simulations, the walls are assumed to have simply supported boundary conditions; the accuracy of this assumption has been experimentally confirmed [21]. More details concerning the Laboratory can be found in [23].

The hybrid mTMM-SEA predictions are also compared to the results of other numerical methods, such as the wave based method (WBM) [23] and the hybrid wave-based transfer matrix method (WB-TMM) [9]. These methods take both the room and wall modes into account. A comparison is made with the hybrid FE-SEA method, that has been discussed earlier in $[15,21]$. Numerical results of the conventional TMM and the TMM using a simplified spatial windowing technique (FTMM) [8] as finite size correction are also shown. Comparisons with alternative mTMM approaches (i.e., other than hybrid mTMM-SEA) are not performed as these will be influenced mainly by the choice of the method for computing the modal radiation impedance. It was already shown in section 2.3 that the wavelet approach for computing the modal radiation impedance, which is employed the conventional hybrid method [15], is the most accurate one.

\subsection{Steel plate}

As a first application, the sound transmission loss of a steel plate is computed with the hybrid mTMM-SEA approach outlined above. The steel plate has a density $\rho=7750 \frac{\mathrm{kg}}{\mathrm{m}^{3}}$, Young's modulus $E=200 \mathrm{GPa}$, Poisson's ratio $\nu=0.28$ and dimensions $1.25 \mathrm{~m} \times 1.50 \mathrm{~m} \times 0.002 \mathrm{~m}$. The experimentally determined damping loss factor of the mounted plate can be found in table 2 . In the complete frequency range of interest, the steel plate can be considered as thin since the bending wavelength is larger than 6 times the thickness of the plate. Consequently, the analytic dynamic stiffness matrix of a thin plate (22-23) can also be used in the hybrid deterministic-SEA approach; the resulting model is termed the hybrid analytic-SEA model (AN-SEA).

Fig. 4 compares the AN-SEA and mTMM-SEA predictions with each other. There is an excellent agreement over the whole frequency range, which confirms that both approaches are equivalent for thin, simply supported plates. The hybrid mTMM-SEA results are also compared with the narrow-band (1/48 octave) transmission loss measurements that have been performed at the 


\begin{tabular}{|l|cccccccccccc|}
\hline$f[\mathrm{~Hz}]$ & 50 & 63 & 80 & 100 & 125 & 160 & 200 & 250 & 315 & 400 & 500 & $\ldots$ \\
\hline$\eta_{\text {steel }}[\%]$ & 5.5 & 5.1 & 4.4 & 4.1 & 3.2 & 3.1 & 2.8 & 2.7 & 2.8 & 3.0 & 3.2 & $\ldots$ \\
\hline$\eta_{\text {PMMA }[\%]}[10.4$ & 8.6 & 6.2 & 8.8 & 6.6 & 6.4 & 6.0 & 5.9 & 5.8 & 6.0 & 5.7 & $\ldots$ \\
\hline$\eta_{\text {brick }}[\%]$ & 3.8 & 6.7 & 7.0 & 4.6 & 4.3 & 3.9 & 5.3 & 3.5 & 1.7 & 2.4 & 2.2 & $\ldots$ \\
\hline$\eta_{\text {glass, } 6 \mathrm{~mm}}[\%]$ & 3.1 & 2.3 & 2.6 & 2.1 & 1.9 & 1.7 & 1.8 & 2.2 & 2.2 & 2.6 & 2.8 & $\ldots$ \\
\hline$\eta_{\text {glass,8mm }[\%]}[\% .4$ & 3.5 & 3.0 & 2.7 & 2.7 & 2.4 & 2.2 & 2.3 & 2.3 & 3.0 & 2.9 & $\ldots$ \\
\hline \hline$f[\mathrm{~Hz}]$ & $\ldots$ & 630 & 800 & 1000 & 1250 & 1600 & 2000 & 2500 & 3150 & 4000 & 5000 & \\
\hline$\eta_{\text {steel }}[\%]$ & $\ldots$ & 3.2 & 3.1 & 3.2 & 3.1 & 3.1 & 2.6 & 2.2 & 1.9 & 1.6 & 1.4 \\
\hline$\eta_{\text {PMMA }}[\%]$ & $\ldots$ & 5.7 & 6.1 & 6.2 & 6.5 & 5.6 & 4.9 & 5.9 & 6.0 & 5.4 & 4.7 & \\
\hline$\eta_{\text {brick }}[\%]$ & $\ldots$ & 3.2 & 2.1 & 1.5 & 2.3 & 1.4 & 1.2 & 1.4 & 0.7 & 0.9 & 0.8 & \\
\hline$\eta_{\text {glass,6mm } 6 \%]}[\%]$ & $\ldots$ & 2.7 & 2.0 & 1.6 & 1.7 & 1.6 & 1.7 & 1.1 & 3.4 & 2.7 & 2.2 & \\
\hline$\eta_{\text {glass }, 8 \mathrm{~mm}}[\%]$ & $\ldots$ & 2.6 & 2.0 & 1.9 & 1.6 & 1.7 & 2.0 & 4.0 & 4.3 & 3.4 & 2.3 & \\
\hline
\end{tabular}

Table 2: Measured damping loss factors $\eta$ for the partition walls tested in the KU Leuven Laboratory of Acoustics.
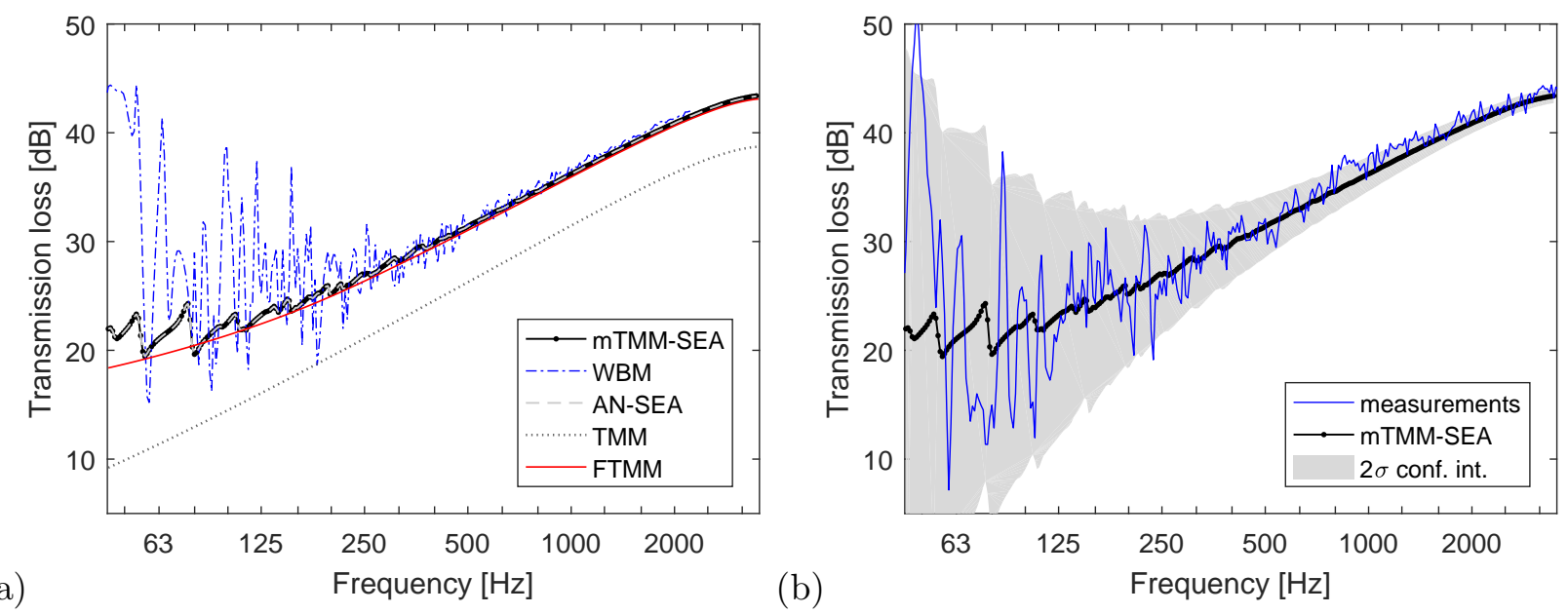

Figure 4: Harmonic sound transmission loss of a steel plate (a) as predicted with the hybrid mTMM-SEA approach, the hybrid WBM approach, the hybrid analytical-SEA approach, the TMM, the FTMM and (b) as measured in the laboratory. The $2 \sigma$ confidence interval, computed with the hybrid mTMM-SEA, is shown in the right figure.

KU Leuven Acoustics Laboratory and with the results of a WBM model. Below the Schroeder frequency [30] of the rooms, which is around $263 \mathrm{~Hz}$, there is a strong oscillation in the measured sound transmission loss and the results of the WBM, since individual room modes influence these results. Above the Schroeder frequency, the measured transmission loss values are close to the mean mTMM-SEA predictions. The $95 \%$ confidence interval of the mTMM-SEA predictions is also plotted. As explained earlier, the related uncertainty is due to the assumption of a diffuse field in the rooms. This uncertainty is well captured by the mTMM-SEA predictions: at low frequencies, where the modal overlap of the rooms is low and their modal behavior has a large influence on the transmission loss, the mTMM-SEA predictions have a large uncertainty, while they have a small uncertainty at high frequencies, where the modal overlap of the rooms is large. 

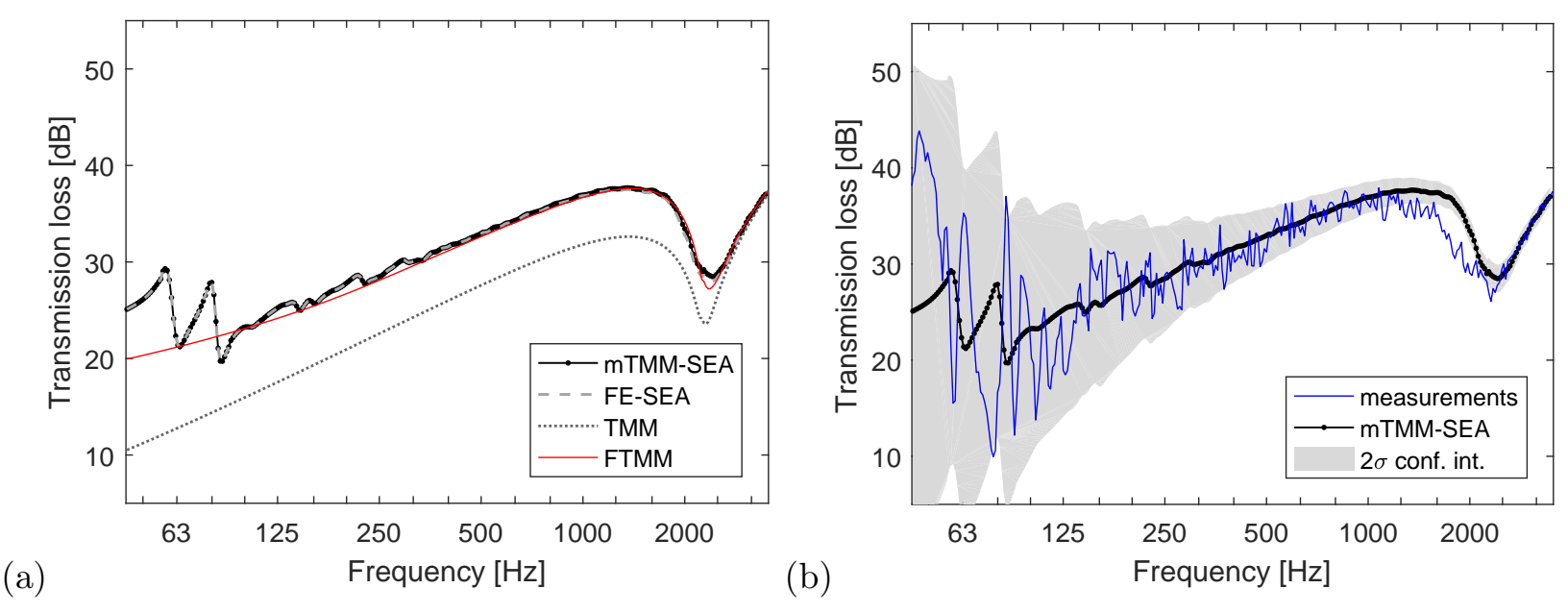

Figure 5: Harmonic sound transmission loss of a PMMA panel (a) as predicted with the hybrid mTMM-SEA approach, the hybrid FE-SEA approach, the conventional TMM, the FTMM, and (b) as measured in the laboratory. The $2 \sigma$ confidence interval, computed with the hybrid mTMM-SEA, is shown in the right figure.

\begin{tabular}{|l|c|c|c|}
\hline Time [s] & mTMM-SEA & FE-SEA & FTMM \\
\hline PMMA panel & 310.1 & 812.9 & 59.7 \\
\hline Brick wall & 71.5 & 5651.5 & 42.9 \\
\hline
\end{tabular}

Table 3: Computation time of the mean sound transmission loss for the hybrid mTMM-SEA method,the FE-SEA method and the FTMM.

\subsection{PMMA panel}

The PMMA panel, considered in this second application, has a density $\rho=1275 \frac{\mathrm{kg}}{\mathrm{m}^{3}}$, Young's modulus $E=4.5 \mathrm{GPa}$, Poisson's ratio $\nu=0.35$ and dimensions $1.25 \mathrm{~m} \times 1.50 \mathrm{~m} \times 0.015 \mathrm{~m}$. The experimentally determined damping loss factor of the mounted panel can be found in table 2 .

Fig. 5 compares the hybrid mTMM-SEA method with the results of a hybrid FE-SEA model. In the hybrid FE-SEA model, the panel is modeled with 4-node shell linear elastic finite elements (of the SHELL181 element type in ANSYS), which can be used for analyzing thin to moderately thick shell structures. Thickness effects cannot be neglected in this example due to the shear deformation and rotational inertia that occur near the coincidence dip, which is situated around $2500 \mathrm{~Hz}$. The displacements at the boundary are restrained to simulate simply supported boundary conditions. It can be concluded from the results that both hybrid methods agree well. The hybrid mTMMSEA results are also compared against narrow-band (1/48 octave) transmission loss measurements $[15,23]$. The predictions of the mTMM-SEA generally agree well with the experimental data, yet the transmission loss below the coincidence dip is slightly overestimated. As argued in [23], this is probably due to the niche which is present in the experiment but not accounted for in the prediction model. The hybrid mTMM-SEA predictions are also compared against conventional TMM predictions, which do not account for the finite dimensions nor the modal behavior of the plate. A conventional TMM model clearly underestimates the sound transmission loss of the plate below coincidence. Above coincidence, the conventional TMM predictions coincide with the hybrid mTMM-SEA predictions, which indicates that at high frequencies, neither the finite dimensions 
nor the modal behavior of the plate influence the sound transmission loss. On the other hand, the FTMM agrees well with the mTMM-SEA predictions, except at lower frequencies where the modal behavior of the plate is important.

The computation times for the PMMA panel using different methods are mentioned in table 3. The fastest method to calculate the mean sound transmission loss is the FTMM which uses a simplified spatial windowing technique. The hybrid methods are more time consuming, but are at the same time also capable of computing the variance and the modal behavior of the plate. As expected, the mTMM-SEA method is more efficient than the FE-SEA method.

\subsection{Hollow brick wall}

In the third application, a perforated brick wall with dimensions $3.25 \mathrm{~m} \times 2.95 \mathrm{~m} \times 0.19 \mathrm{~m}$ is considered. The wall is plastered at both sides. The acoustic behavior of perforated brick walls is complex, given the inhomogeneities at three different scales: the fire clay material, the brick where small cavities are present in the fire clay because of the perforations, and the entire wall where the bricks are held together by mortar layers. When the inhomogeneities are small compared to the wavelength, and when the stiffness is not too different in different directions, the wall can be modeled as homogeneous and isotropic. The thickness effects, however, can not be neglected: not only is shear deformation important, thickness resonances (i.e., Lamb modes) are often observed in the audio frequency range [31]. Following [9], the equivalent Young's modulus, Poisson's ratio, density and thickness are taken as $E=1825 \mathrm{MPa}, \nu=0.2, \rho=613.5 \frac{\mathrm{kg}}{\mathrm{m}^{3}}$ and $t=0.2934 \mathrm{~m}$, respectively. The experimentally determined damping loss factor of the wall in the transmission opening can be found in table 2 .

Fig. 6 shows the predictions of the hybrid mTMM-SEA method, of the conventional TMM, of the FTMM and of a hybrid FE-SEA model. In the hybrid FE-SEA model, the wall is modeled using 8-node solid linear elastic finite elements (of the SOLID45 element type in ANSYS) and only the boundary displacements in the middle plane of the wall are restrained. The hybrid mTMM-SEA and FE-SEA predictions agree well but they are not exactly the same, which is expected since the
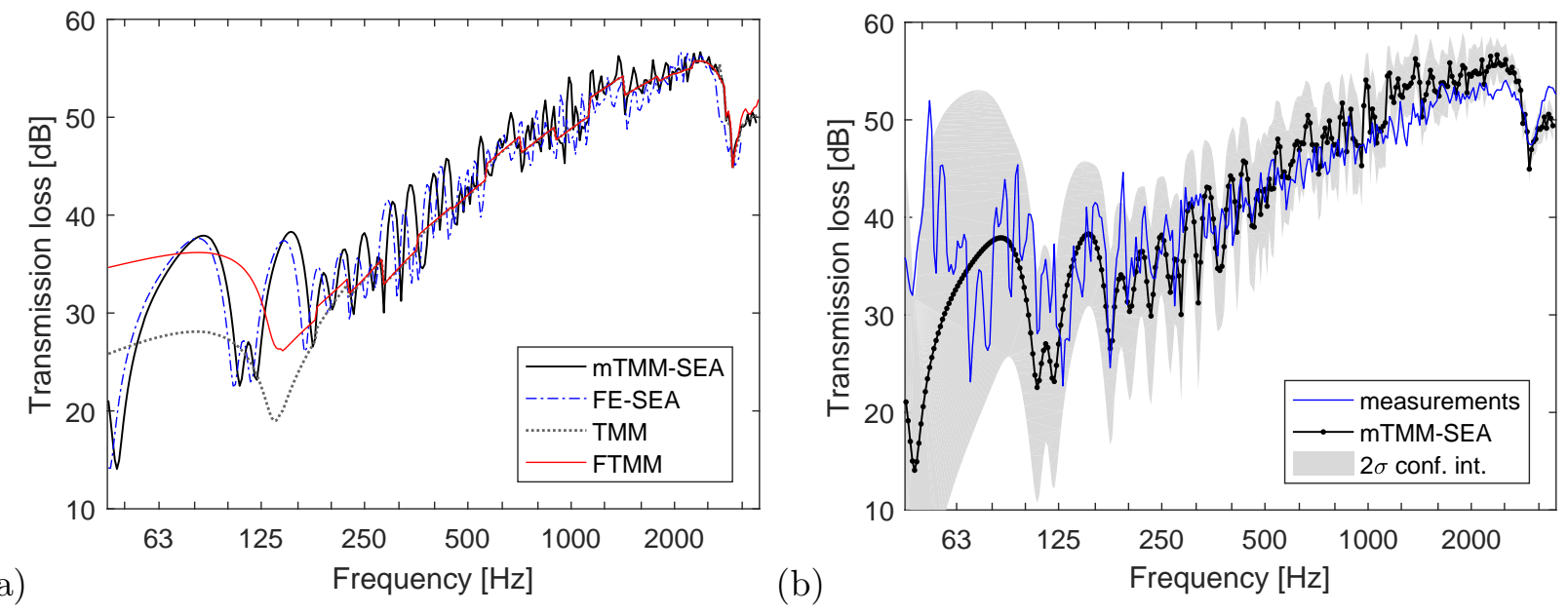

Figure 6: Harmonic sound transmission loss of a perforated brick wall (a) as predicted with the hybrid mTMM-SEA approach, the hybrid FE-SEA approach, the conventional TMM, the FTMM, and (b) as measured in the laboratory. The $2 \sigma$ confidence interval, computed with the hybrid mTMM-SEA, is shown in the right figure. 
boundary conditions are slightly different and since (27) results in an approximation of the true wall behavior for thick walls. Both models correctly predict the first thickness resonance of the wall at around $3000 \mathrm{~Hz}$. The comparison with the conventional TMM and the FTMM predictions illustrates that the modal behavior of the wall plays an important role up to about $500 \mathrm{~Hz}$. The low modal density of the wall makes that the coincidence dip, which can be observed in the conventional TMM and the FTMM predictions between $125 \mathrm{~Hz}$ and $160 \mathrm{~Hz}$, cannot develop. This is confirmed by the comparison of the hybrid mTMM-SEA results with measured values [15, 23]. Again a good agreement is observed between the measurements and the mTMM-SEA predictions.

The computation times are reported in Table 3. The computation cost of the mTMM-SEA model is much lower than for the FE-SEA model in this case, because (1) the finite element model of the wall is a $3 \mathrm{D}$ solid model, and (2) the modal density of the wall is low. The FTMM results in only half of the computation time of the mTMM-SEA approach, but shows deviations at lower frequencies.

\subsection{Sandwich panel}

The fourth system concerns a sandwich panel of dimensions $1.25 \mathrm{~m} \times 1.50 \mathrm{~m} \times 0.150 \mathrm{~m}$. The panel consists of a core of expanded polystyrene (EPS) to which a $4 \mathrm{~mm}$ thick fiberboard panel is glued at each side. In the simulations, the fiberboard has a density $\rho=765 \frac{\mathrm{kg}}{\mathrm{m}^{3}}$, Young's modulus $E=3.5 \mathrm{GPa}$, Poisson's ratio $\nu=0.46$ and loss factor $\eta=0.01$, while the EPS core, which is modeled as an elastic layer, has a density $\rho=20 \frac{\mathrm{kg}}{\mathrm{m}^{3}}$, Young's modulus $E=12 \mathrm{MPa}$, Poisson's ratio $\nu=0.10$ and loss factor $\eta=0.05$.

Fig. 7 compares the hybrid mTMM-SEA predictions with conventional TMM and FTMM predictions and predictions using a hybrid WB-TMM model.The hybrid mTMM-SEA results are also compared with measured values [15]. All models correctly predict the resonances around $1200 \mathrm{~Hz}$ and around $3300 \mathrm{~Hz}$, which are dilation and thickness resonance dips, respectively [9]. Below the dilation resonance frequency, the hybrid WB-TMM and mTMM-SEA models generally yield slightly higher transmission loss predictions than the conventional TMM model, while the FTMM
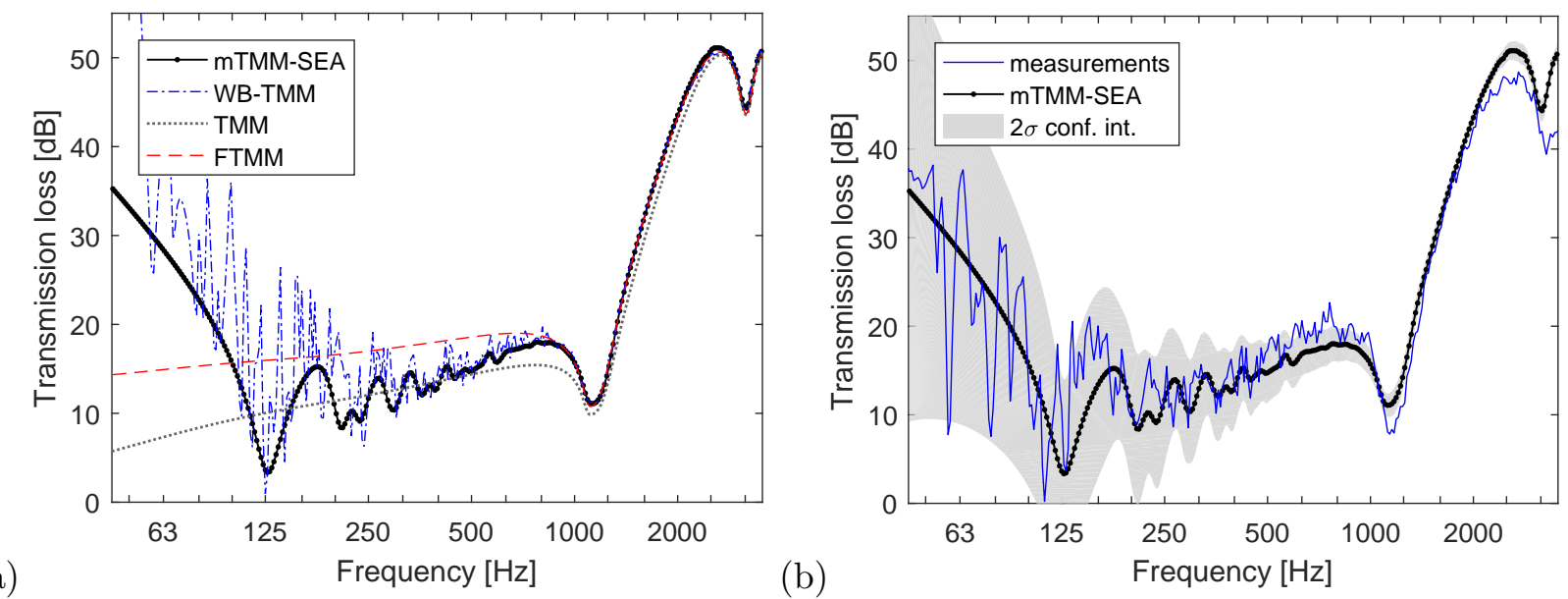

Figure 7: Harmonic sound transmission loss of a sandwich panel (a) as predicted with the hybrid mTMM-SEA approach, the hybrid WB-TMM approach, the conventional TMM, the FTMM and (b) as measured in the laboratory. The $2 \sigma$ confidence interval, computed with the hybrid mTMM-SEA, is shown in the right figure. 
overestimates the sound transmission loss in comparison to the other models. Above the dilation resonance frequency, each model predicts the same transmission loss, which indicates that neither the finite size of the wall, nor individual wall resonances, nor individual room resonances influence the transmission loss at these high frequencies.

The dip caused by the fundamental panel resonance, which is at around $125 \mathrm{~Hz}$, and the transition from stiffness to mass behavior at this point are clearly visible in the hybrid mTMM-SEA predictions. Because of the high stiffness to mass ratio, the sound transmission loss of the sandwich panel increases strongly with decreasing frequency below the fundamental mode [32]. The conventional TMM and FTMM do not capture this stiffness effect which explains the large difference. The hybrid predictions also agree well with the measurements, although they slightly underestimate the transmission loss below the dilation resonance.

\subsection{Double-leaf wall}

In this section a double-leaf wall, consisting of a steel plate, a mineral wool core with a flow resistivity of $34 \mathrm{kNs} / \mathrm{m}^{4}$ and a laminate plate, are considered. The different layers are glued onto each other. This validation example was adopted from the literature, as it is known to be a challenging example for modeling poro-elastic layers. The material properties and experimental results in $1 / 3$ octave bands can be found in [4].

The mineral wool is a poro-elastic material. It may be modeled as an equivalent fluid, e.g., using the empirical model of Delany and Bazley [33]. In this model, the complex characteristic impedance $Z_{c}$ and the propagation coefficient $\Gamma=\mathrm{i} k$ are derived from the flow resistivity. These complex parameters are then implemented in the transfer matrix of a fluid as explained in [19]. The advantage is that only the flow resistivity $\sigma$ and thickness of the porous layer have to be known as input parameters. When using an equivalent fluid model, it is assumed that the frame is motionless and that only a compressional wave will travel through the layer. However, in case of a poro-elastic material with a flexible frame a more elaborated model may be needed such as the Biot model [34, 35]. This model has been incorporated into the transfer matrix method [3].
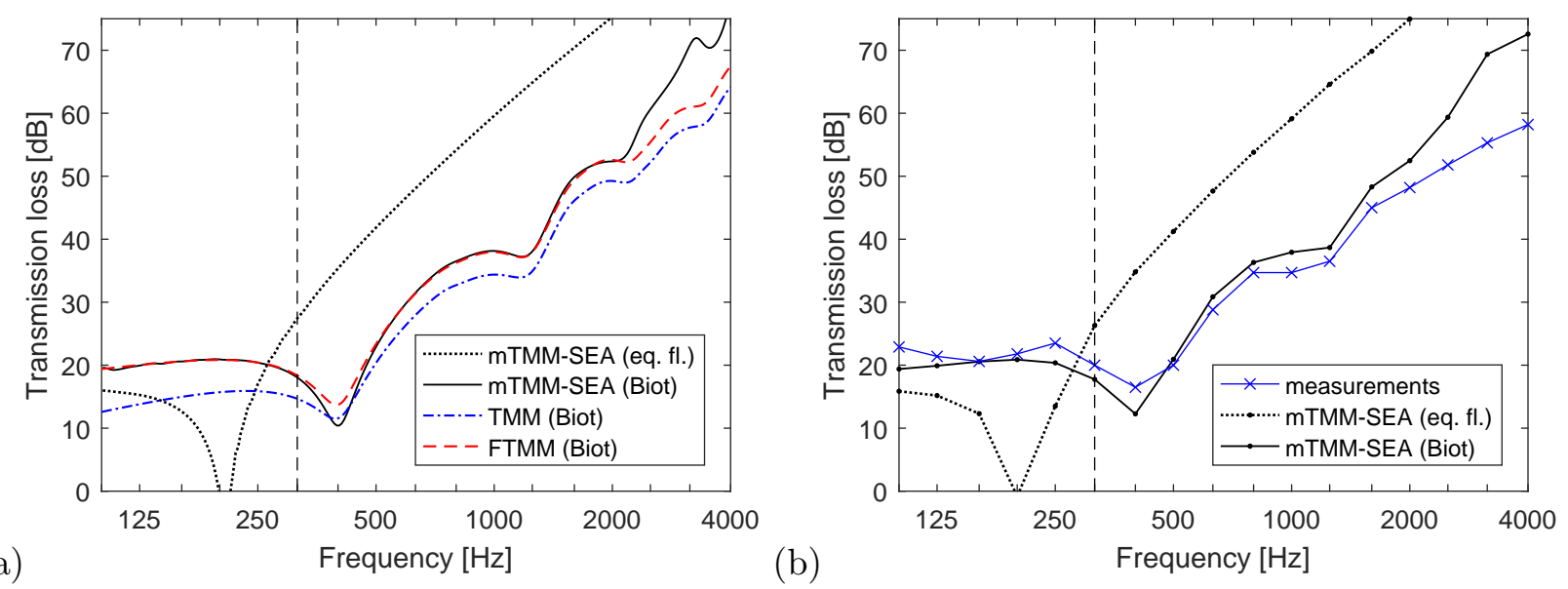

Figure 8: Harmonic sound transmission loss of a double-leaf wall with poro-elastic material in the cavity (a) as predicted with the hybrid mTMM-SEA approach using an equivalent fluid model or a Biot model, the conventional TMM, the FTMM and (b) as measured in [4]. The results in the right figure are band-averaged to $1 / 3$ octave bands. 

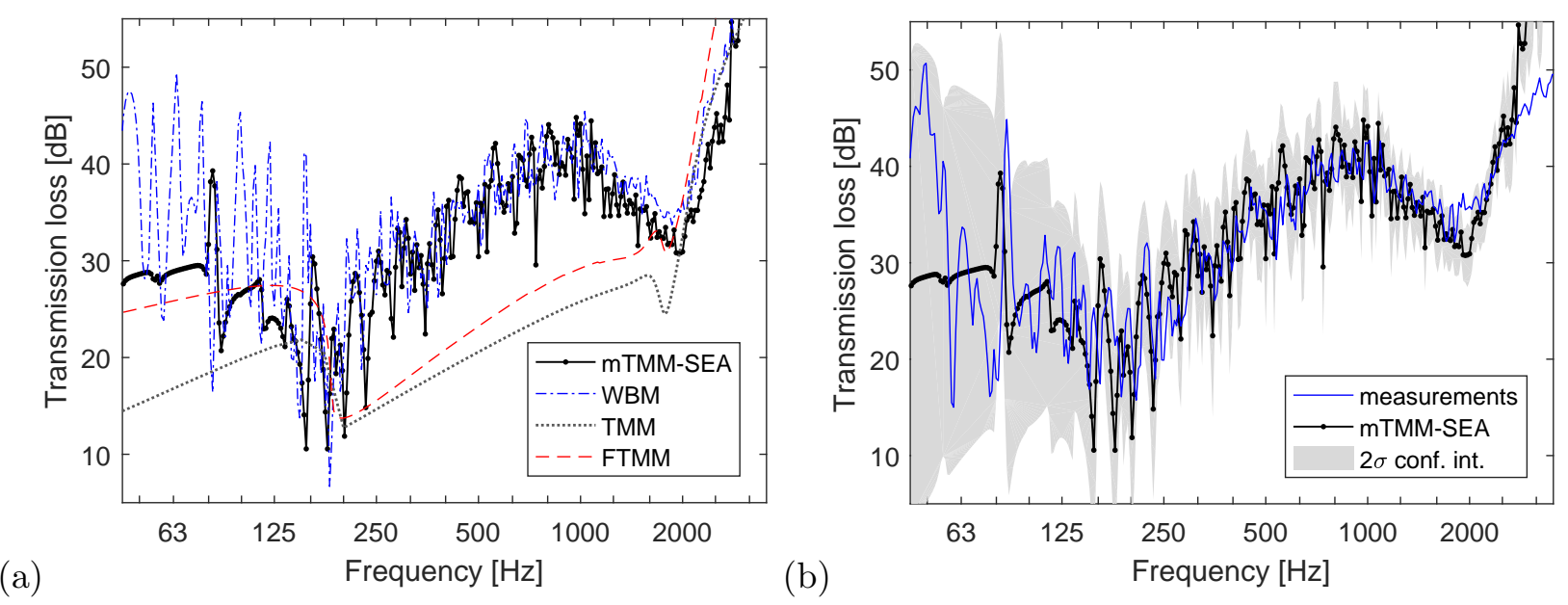

Figure 9: Harmonic sound transmission loss of a double glazing (a) as predicted with the hybrid mTMM-SEA approach, the hybrid WBM approach, the conventional TMM, the FTMM and (b) as measured in the laboratory. The $2 \sigma$ confidence interval, computed with the hybrid mTMM-SEA, is shown in the right figure.

While the Biot model is physically more accurate, it requires also more input parameters than the Delany-Bazley equivalent fluid model.

The Biot model and the equivalent fluid model are implemented in the TMM framework, allowing to examine possible differences. Numerical predictions of the TMM, FTMM and mTMMSEA approach using a Biot model and results of the mTMM-SEA approach using an equivalent fluid model are displayed in Fig. 8. The band-averaged results of the mTMM-SEA approach using both models for the poro-elastic material, are also compared to experimental data. Note that the empirical formulae of Delany and Bazley [33] are only valid in the range $0.01 \leqslant \rho_{a} f / \sigma \leqslant 1$, which implies that the equivalent fluid model is valid from $315 \mathrm{~Hz}$ onwards. This is indicated by the vertical dashed line. A comparison with the experimental results shows that the equivalent model overestimates clearly the sound transmission loss in this example, unlike the Biot model, which is able to capture the behavior of the mineral wool accurately.

\subsection{Double glazing}

The final structure is a double glazing with dimensions $1.25 \mathrm{~m} \times 1.50 \mathrm{~m} \times 0.026 \mathrm{~m}$ which consists of two glass panels with a thickness of $6 \mathrm{~mm}$ and $8 \mathrm{~mm}$, separated by an air cavity of $12 \mathrm{~mm}$. For the simulations, the following material properties for the glass panels are taken: density $\rho=2500 \frac{\mathrm{kg}}{\mathrm{m}^{3}}$, Young's modulus $E=62 \mathrm{GPa}$ and Poisson's ratio $\nu=0.24$. The experimentally determined damping loss factor of both glass panes in mounted condition can be found in table 2. The air cavity is taken to be undamped.

As discussed earlier, the mTMM-SEA approach cannot be used for estimating the sound insulation of layered walls containing both solid layers and undamped air layers. However, it is possible to model the glazing using a Rayleigh-Ritz approach, in which the air layer is modeled analytically, as a hard-walled cavity, while the glass panels are modeled as elastic thin plates using the mTMM. The resulting double glazing model is then again coupled to SEA models, describing the neighboring rooms. The adopted equations are provided in Appendix A.

Fig. 9 displays the predictions of this modified mTMM-SEA method in comparison with results of the conventional TMM and of a WBM model. The results are also compared with measured 
values [15]. All models agree well, except for the conventional TMM and the FTMM which largely underestimate the sound transmission loss in between the mass-spring-mass resonance (around $160-200 \mathrm{~Hz}$ ) and the coincidence dip (around $1600-2000 \mathrm{~Hz}$ ), as the empty cavity was modeled using a fluid layer in these approaches. Note that the WBM accounts for the modal behavior of the rooms, and it is therefore computationally much more expensive than the hybrid mTMM-SEA. Above $2500 \mathrm{~Hz}$ the predictions overestimate the sound transmission loss in comparison with the measurements; this is probably due to the structural coupling between the edges of the glass panels at in the measurement setup, which is not accounted for in the prediction models.

\section{Conclusions}

A hybridization between the transfer matrix method and statistical energy analysis has been developed for computing the diffuse sound transmission through finite walls and floors that consist of layers of solid, fluid and/or poroelastic material. The modal behavior of the wall is approximately accounted for. The sound insulation predictions for different examples have been compared with experimental data. An overall good agreement has been observed. A comparison with alternative methods yields the following conclusions: The approach is computationally very efficient when compared to the hybrid FE-SEA approach since detailed finite element modeling of the wall is avoided, while maintaining the same accuracy. The connection with statistical energy analysis also enables to estimate the variance of the predicted sound insulation that is inherent to the statistical diffuse field models of the rooms. This uncertainty characterization is not possible when using TMM-based methods. Additionally, the wavelet approach in combination with the hybrid framework allows to incorporate cross-modal coupling in the mTMM-SEA approach, which was shown to be important at low to medium frequencies, especially at anti-resonance frequencies of the wall. In terms of future developments, the present analysis could be extended to include more general boundary conditions, baffle conditions, and curved wall systems. For example, if unbaffled or partially baffled rather than baffled conditions are of interest, the analysis of Section 2.3 could be modified along the lines of [25].

\section{Acknowledgements}

This research was supported by a PhD Fellowship (C. Decraene) and Postdoctoral Fellowships (E. Reynders and A. Dijckmans) from the Research Foundation - Flanders, and by an Assistant Professorship from KU Leuven (E. Reynders). The financial support of FWO and KU Leuven is gratefuly acknowledged.

\section{Appendix A: Double glazing model}

The deterministic dynamic stiffness matrix of the double glazing which consists of two glass panels with dimensions $L_{x} \times L_{y}$ and a cavity with dimensions $l_{x} \times l_{y} \times l_{z}$, is obtained using a Rayleigh-Ritz model. The different elements of the matrix are deduced by examining the individual components and their interaction. First, the dynamic stiffness matrix of the cavity is discussed in more detail. Because the cavity is an acoustic component, pressure is used as the physical variable instead of displacements which is used for structural components. The pressure in the acoustic 
cavity can be approximated using a finite set of $N_{r}$ basis functions $\psi$ and corresponding generalized coordinates $b$ :

$$
p(\mathbf{x}, z, \omega) \approx \sum_{r=1}^{N_{r}} \psi_{r}(\mathbf{x}, z) b_{r}(\omega)
$$

The basis functions $\psi$ are chosen to be the mode shapes of a hard-walled rectangular cavity, normalized to unit acoustic mass:

$$
\psi_{r}(\mathbf{x}, z)=c_{a} \sqrt{\frac{\epsilon_{x} \epsilon_{y} \epsilon_{z}}{l_{x} l_{y} l_{z}}} \cos \left(\frac{n_{x} \pi x}{l_{x}}\right) \cos \left(\frac{n_{y} \pi y}{l_{y}}\right) \cos \left(\frac{n_{z} \pi z}{l_{z}}\right)
$$

where $n_{x}, n_{y}, n_{z}$ are the integer number of half wavelengths in the $\mathrm{x}$-, $\mathrm{y}$ - and z-directions of mode $r . \epsilon_{x}, \epsilon_{y}$ and $\epsilon_{z}$ are integers equal to one when $n_{x}, n_{y}$ and $n_{z}$ equal zero and equal to two otherwise. The corresponding natural frequencies are:

$$
\omega_{\mathrm{cav}}=c_{a} \sqrt{\left(\frac{n_{x} \pi}{l_{x}}\right)^{2}+\left(\frac{n_{y} \pi}{l_{y}}\right)^{2}+\left(\frac{n_{z} \pi}{l_{z}}\right)^{2}}
$$

The mass and stiffness matrices are then easily computed for a hard-walled rectangular room as the generalized coordinates are the modal coordinates of the cavity. Combining the mass and stiffness matrix results in the dynamic stiffness matrix of the cavity:

$$
D_{\text {cav }, r s}=\left(-\omega^{2}+\omega_{\text {cav }}^{2}\left(1+\mathrm{i} \eta_{\text {cav }}\right)\right) \delta_{r s}
$$

where $r, s$ represent the element of row $r$ and column $s$ in the matrix. Because the damping in the air cavity is not considered in this example, the damping coefficient $\eta_{\text {cav }}$ is equal to zero. In the next step the interaction of the glass panels with the cavity is elaborated. Inserting equations (A.1) and (A.4) into Lagrange's equations of motion yields the following result as the cavity is excited by the movements of two glass panels:

$$
\mathbf{D}_{\text {cav }} \mathbf{q}_{\mathrm{cav}}=\mathbf{Q}_{\mathrm{cav}, 1} \mathbf{q}_{\mathrm{glass} 1}+\mathbf{Q}_{\mathrm{cav}, 2} \mathbf{q}_{\mathrm{glass} 2}
$$

with

$$
\mathbf{Q}_{\text {cav }, i, r} \mathbf{q}_{\text {glass } i}=-\int_{S} \rho_{a} \ddot{\mathbf{u}}^{\mathrm{T}} \mathbf{n}_{S} \psi_{r} \mathrm{~d} S
$$

$\mathbf{q}_{\mathrm{cav}}$ includes the modal degrees of freedom of the hard-walled cavity $b$, while $\mathbf{q}_{\text {glass1 }}$ and $\mathbf{q}_{\text {glass2 }}$ collect the generalized degrees of freedom of the glass panels $q . \mathbf{Q}_{\mathrm{cav}, i}$ determines the structurefluid interaction with $r$ representing row $r$ of matrix $\mathbf{Q}_{\text {cav }, i}$, $\mathbf{u}$ the displacement of the surface $S$ of the structure and $\mathbf{n}_{S}$ the normal on the surface $S$ pointing towards the acoustic fluid. This expression can be further expanded by substituting equations (1) and (21) into the displacements $\mathbf{u}$. Consequently, the structure-fluid interaction $\mathbf{Q}_{\mathrm{cav}, i}$ can be constructed by applying the following formula for each mode $r=\left(n_{x}, n_{y}, n_{z}\right)$ of the cavity with each mode $s=\left(p_{x}, p_{y}\right)$ of the imposed basis function on the glass panel $i$.

$$
Q_{\mathrm{cav}, i, r s}=\omega^{2} c_{a} l_{x}^{2} L_{x} l_{y}^{2} L_{y} p_{x} p_{y} \sqrt{\frac{\epsilon_{x} \epsilon_{y} \epsilon_{z}}{l_{x} l_{y} l_{z}}} \rho_{a} \frac{\left(1-(-1)^{p_{x}} \cos \left(\frac{n_{x} \pi L_{x}}{l_{x}}\right)\right)\left(1-(-1)^{p_{y}} \cos \left(\frac{n_{y} \pi L_{y}}{l_{y}}\right)\right)}{\pi^{2}\left(l_{x}^{2} p_{x}^{2}-L_{x}^{2} n_{x}^{2}\right)\left(l_{y}^{2} p_{y}^{2}-L_{y}^{2} n_{y}^{2}\right)}
$$


In a similar way the dynamic stiffness matrix of the glass panels which are treated as thin mTMM layers in this model, and their interaction with the acoustic cavity is shortly summarized. The dynamic stiffness matrix $\mathbf{D}_{\text {glass }}$ of the glass panels is determined by the mTMM as seen in section 2.2. The forces acting on the two glass panels have to be in equilibrium:

$$
\begin{aligned}
& \mathbf{D}_{\text {glass } 1} \mathbf{q}_{\text {glass } 1}=\mathbf{Q}_{1, \text { cav }} \mathbf{q}_{\text {cav }}+\mathbf{f}_{\text {glass1 }} \\
& \mathbf{D}_{\text {glass } 2} \mathbf{q}_{\text {glass } 2}=\mathbf{Q}_{2, \text { cav }} \mathbf{q}_{\text {cav }}+\mathbf{f}_{\text {glass2 }}
\end{aligned}
$$

The fluid-structure interaction $\mathbf{Q}_{i, \text { cav }}$ can be derived of the structure-fluid interaction $\mathbf{Q}_{\text {cav }, i}$.

$$
\mathbf{Q}_{i, \mathrm{cav}}=-\frac{\mathbf{Q}_{\mathrm{cav}, i}^{\mathrm{T}}}{\rho_{a}}
$$

Equation (A.5) can be solved for $\mathbf{q}_{\mathrm{cav}}$. The obtained expression can then be used in equations (A.8) and (A.9). In this way the global deterministic dynamic stiffness matrix $\mathbf{D}_{\mathrm{d}}$ of the double glazing with amplitude vector $\mathbf{q}=\left[\begin{array}{l}\mathbf{q}_{\text {glass } 1} \\ \mathbf{q}_{\text {glass } 2}\end{array}\right]$ and load vector $\mathbf{f}=\left[\begin{array}{l}\mathbf{f}_{\text {glass } 1} \\ \mathbf{f}_{\text {glass } 2}\end{array}\right]$ can be derived:

$$
\begin{aligned}
& \mathbf{D}_{\mathrm{d}} \mathbf{q}=\mathbf{f} \\
& \mathbf{D}_{\mathrm{d}}=\left[\begin{array}{cc}
\mathbf{D}_{\text {glass } 1}-\mathbf{Q}_{1, \mathrm{cav}} \mathbf{D}_{\text {cav }}^{-1} \mathbf{Q}_{\mathrm{cav}, 1} & -\mathbf{Q}_{1, \mathrm{cav}} \mathbf{D}_{\mathrm{cav}}^{-1} \mathbf{Q}_{\mathrm{cav}, 2} \\
-\mathbf{Q}_{2, \mathrm{cav}} \mathbf{D}_{\text {cav }}^{-1} \mathbf{Q}_{\mathrm{cav}, 1} & \mathbf{D}_{\text {glass } 2}-\mathbf{Q}_{2, \mathrm{cav}} \mathbf{D}_{\text {cav }}^{-1} \mathbf{Q}_{\mathrm{cav}, 2}
\end{array}\right]
\end{aligned}
$$

\section{References}

[1] W. Lauriks, P. Mees, J. F. Allard, The acoustic transmission through layered systems, J. Sound Vibr. 155 (1992) 125-132.

[2] B. Brouard, D. Lafarge, J. F. Allard, A general method of modelling sound propagation in layered media, J. Sound Vibr. 183 (1995) 129-142.

[3] J. F. Allard, N. Atalla, Propagation of sound in porous media, second ed., John Wiley \& Sons, Chichester, UK, 2009.

[4] M. Villot, C. Guigou, L. Gagliardini, Predicting the acoustical radiation of finite size multi-layered structures by applying spatial windowing on infinite structures, J. Sound Vibr. 245 (2001) 433-455.

[5] L. Cremer, M. Heckl, B. A. T. Petersson, Structure-borne sound: Structural vibrations and sound radiation at audio frequencies, third ed., Springer, Berlin, 2005.

[6] D. Rhazi, N. Atalla, A simple method to account for size effects in the transfer matrix method, J. Acoust. Soc. Am., Express Letters 127 (2010) 30-36.

[7] P. Bonfiglio, F. Pompoli, R. Lionti, A reduced-order integral formulation to account for the finite size effect of isotropic square panels using the transfer matrix method, J. Acoust. Soc. Am. 139 (2016) 1773-1783.

[8] T. E. Vigran, Predicting the sound reduction index of finite size specimen by a simplified spatial windowing technique, J. Sound Vibr. 325 (2009) 507-512.

[9] A. Dijckmans, G. Vermeir, Development of a hybrid wave based - transfer matrix model for sound transmission analysis, J. Acoust. Soc. Am. 133 (2013) 2157-2168.

[10] D. Rhazi, N. Atalla, Acoustic and vibration response of a structure with added noise control treatment under various excitations, J. Acoust. Soc. Am. 135 (2014) 693-704.

[11] R. H. Lyon, Needed: a new definition of diffusion, J. Acoust. Soc. Am. 56 (1974) 1300-1302.

[12] P. J. Shorter, R. S. Langley, On the reciprocity relationship between direct field radiation and diffuse reverberant loading, J. Acoust. Soc. Am. 117 (2005) 85-95.

[13] P. J. Shorter, R. S. Langley, Vibro-acoustic analysis of complex systems, J. Sound Vibr. 288 (2005) 669-699.

[14] R. S. Langley, V. Cotoni, Response variance prediction for uncertain vibro-acoustic systems using a hybrid deterministic-statistical method, J. Acoust. Soc. Am. 122 (2007) 3445-3463. 
[15] E. Reynders, R. S. Langley, A. Dijckmans, G. Vermeir, A hybrid finite element - statistical energy analysis approach to robust sound transmission modelling, J. Sound Vibr. 333 (2014) 4621-4636.

[16] R. S. Langley, On the diffuse field reciprocity relationship and vibrational energy variance in a random subsystem at high frequencies, J. Acoust. Soc. Am. 121 (2007) 913-921.

[17] R. S. Langley, J. A. Cordioli, Hybrid deterministic-statistic analysis of vibro-acoustic systems with domain couplings on statistical components, J. Sound Vibr. 321 (2009) 893-912.

[18] R. H. Lyon, R. G. DeJong, Theory and application of statistical energy analysis, second ed., ButterworthHeinemann, Newton, MA, 1995.

[19] T. E. Vigran, Building Acoustics, Taylor \& Francis, Oxon, UK, 2008.

[20] V. Cotoni, P. Shorter, R. Langley, Numerical and experimental validation of a hybrid finite element-statistical energy analysis method, J. Acoust. Soc. Am. 122 (2007) 259-270.

[21] E. Reynders, C. Van hoorickx, A. Dijckmans, Sound transmission through finite rib-stiffened and orthotropic plates, Acta Acust. United Acust. 102 (2016) 999-1010.

[22] D. L. Folds, C. D. Loggins, Transmission and reflection of ultrasonic waves in layered media, J. Acoust. Soc. Am. 62 (1977) 1102-1109.

[23] A. Dijckmans, Wave based calculation methods for sound-structure interaction. Application to sound insulation and sound radiation of composite walls and floors, Ph.D. thesis, KU Leuven, 2011.

[24] L. Rayleigh, The theory of sound, second ed., volume II, Dover publications, New York, NY, 1894.

[25] R. S. Langley, Numerical evaluation of the acoustic radiation from planar structures with general baffle conditions using wavelets, J. Acoust. Soc. Am. 121 (2007) 766-777.

[26] F. G. Leppington, E. G. Broadbent, K. H. Heron, The acoustic radiation efficiency of rectangular panels, Proceedings of the Royal Society A - Mathematical, Physical and Engineering Sciences 382 (1982) 245-271.

[27] J. L. Davy, D. J. Larner, R. R. Wareing, J. R. Pearse, The acoustic radiation impedance of a rectangular panel, Build. Environ. 92 (2015) 743-755.

[28] ISO 10140:2010: Acoustics - Laboratory measurement of sound insulation of building elements, International Organization for Standardization, 2010.

[29] ISO 10848-1:2006: Acoustics - Laboratory measurement of the flanking transmission of airborne and impact sound between adjoining rooms - Part 1: Frame document, International Organization for Standardization, 2006.

[30] M. R. Schroeder, The "Schroeder frequency" revisited, J. Acoust. Soc. Am. 99 (1996) 3240-3241.

[31] G. Jacqus, S. Berger, V. Gibiat, P. Jean, M. Villot, S. Ciukay, A homogenised vibratory model for predicting the acoustic properties of hollow brick walls, J. Sound Vibr. 330 (2011) 3400-3409.

[32] F. Fahy, P. Gardonio, Sound and structural vibration: radiation, transmission and response, second ed., Academic Press, Oxford, UK, 2007.

[33] M. E. Delany, E. N. Bazley, Acoustical properties of fibrous absorbent materials, Appl. Acoust. 3 (1970) $105-116$.

[34] M. A. Biot, Theory of propagation of elastic waves in a fluid-saturated porous solid. I. low-frequency range, J. Acoust. Soc. Am. 28 (1956) 168-178.

[35] M. A. Biot, Theory of propagation of elastic waves in a fluid-saturated porous solid. II. high-frequency range, J. Acoust. Soc. Am. 28 (1956) 179-191. 